\title{
Déficit moteur et douleur pluriradiculaire faisant découvrir une maladie de Devic
}

\author{
Neurological Deficiency and Multiradicular Pain Revealing Devic's Disease
}

\author{
F. Balen $\cdot$ S. Charpentier $\cdot$ O. Brunel \\ Reçu le 13 février 2018; accepté le 25 avril 2018 \\ (C) SFMU et Lavoisier SAS 2018
}

\section{Introduction}

La maladie de Devic (ou neuromyélite optique [NMO]) est une pathologie inflammatoire auto-immune démyélinisante sévère et récidivante du système nerveux central, affectant principalement la moelle épinière et les nerfs optiques. Nous rapportons ici le cas d'une patiente présentant une atteinte uniquement médullaire de cette maladie. Le but de cet article est de présenter une pathologie potentiellement invalidante mais curable, méconnue de l'urgentiste car rare.

\section{Observation}

Une patiente de 43 ans sans antécédents notables se présente aux urgences pour douleur invalidante du membre inférieur gauche. La symptomatologie évolue crescendo depuis deux semaines et a motivé une consultation la veille avec introduction d'une antalgie par palier 2 dans l'hypothèse d'une lombosciatique ou d'un zona de localisation atypique sans symptomatologie cutanée. Il n'y a pas de notion de voyage récent, pas de morsure de tique.

À l'arrivée aux urgences, la patiente est apyrétique et présente une douleur intense d'allure neuropathique (sensation de brûlure, d'hypoesthésie puis d'allodynie) de la face antérieure et latérale du membre inférieur gauche. L'existence d'un déficit $3 / 5$ du releveur du pied et une antalgie par morphine insuffisante font réaliser une tomodensitométrie (TDM) du rachis lombaire pour éliminer une urgence neuro-

F. Balen $(\bowtie) \cdot$ S. Charpentier $\cdot$ O. Brunel

Pôle de médecine d'urgence/Samu, CHU de Toulouse,

place du Docteur-Baylac, TSA 40031,

F-31059 Toulouse cedex 09, France

e-mail : balen.f@chu-toulouse.fr

S. Charpentier

Université Toulouse-III-Paul-Sabatier,

route de Narbonne, F-31330 Toulouse, France chirurgicale. La TDM n'étant pas contributive et le déficit moteur s'étendant en proximal, il est décidé de compléter les explorations par une imagerie par résonance magnétique (IRM) médullaire (Fig. 1A). Malgré l'absence de syndrome inflammatoire biologique, une ponction lombaire est réalisée, retrouvant une hyperprotéinorachie à glycorachie normale et une hypercellularité à prédominance lymphocytaire. Les traitements antibiotiques et antiviraux débutés seront arrêtés dans un second temps après rendu négatif des cultures et PCR.

Devant ce tableau de myélite avec atteinte de plus de deux segments, les explorations complémentaires en service de neurologie [1] sont réalisées à la recherche d'un syndrome de Devic. Ces dernières retrouvent des anticorps antiaquaporine 4 (AQP 4) sériques fortement positifs et des anomalies à l'IRM cérébrale (Fig. 1B) (multiples hypersignaux T2 : juxtaventriculaires au niveau des ventricules latéraux et quatrième ventricule, en regard de l'aera postrema et du foramen de Monro droit). Le diagnostic de myélite sévère dans le cadre d'une neuromyélite optique est posé, et une corticothérapie est débutée. Devant l'absence d'efficacité, des échanges plasmatiques sont réalisés, permettant une amélioration des phénomènes douloureux et une récupération partielle du déficit moteur (complété par une prise en charge par kinésithérapie à la sortie de l'hôpital). Un traitement de fond par rituximab est également initié.

\section{Discussion}

D'une prévalence estimée entre 1 et 4,4/100 000 [2], la maladie de Devic constitue une atteinte inflammatoire rare du SNC. Longtemps considérée comme une variante clinique de la sclérose en plaques, les progrès de la biologie moléculaire ont permis de la distinguer de cette dernière [3], en approchant son mécanisme physiopathologique. Son étiologie serait auto-immune par production d'AQP 4 [4], aquaporine 4 retrouvée principalement au niveau des astrocytes 

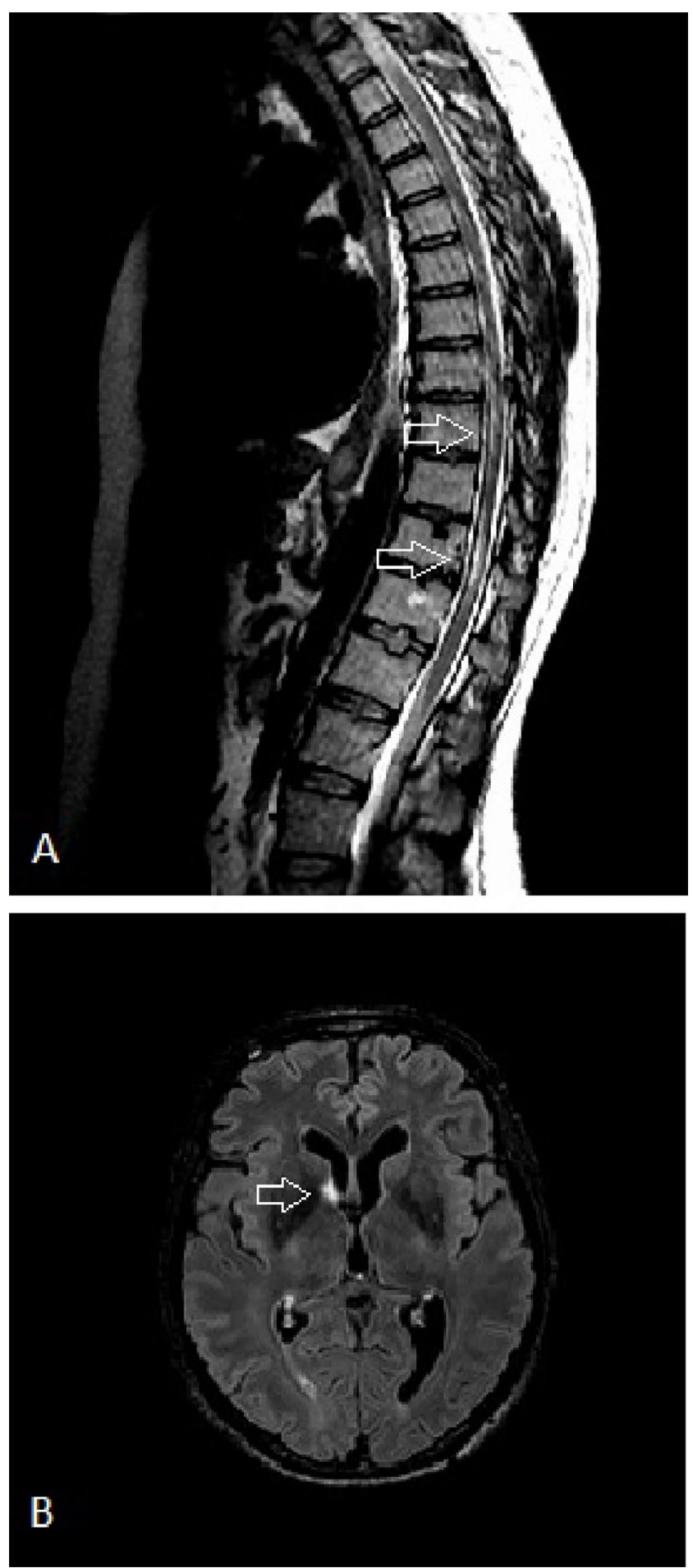

Fig. 1 A. IRM médullaire séquence T2 coupe sagittale : hypersignal médullaire en regard de T9 et T11 (flèches). B. IRM cérébrale séquence FLAIR coupe axiale : hypersignal de la substance blanche juxtaventriculaire droite (flèche) médullaires et du nerf optique. Sur le plan épidémiologique, l'âge médian de survenue est de 39 ans avec une prédominance féminine nette (avec un rapport de neuf cas féminins pour un cas masculin) [2]. La présentation clinique de la maladie de Devic peut associer myélite multiétagée (au moins trois segments), névrite optique récurrente et symptomatologie neurologique aspécifique (nausées/vomissements, narcolepsie et dysfonction neuroendocrine). Des cas de hoquet et de vomissements incoercibles pouvant durer plusieurs jours sont également décrits [5].

Le diagnostic peut être porté sur la présence d'au moins deux des trois critères suivants [6] :

- lésion continue d'une hauteur supérieure à trois segments vertébraux à l'IRM médullaire ;

- absence d'argument pour une sclérose en plaques à l'IRM cérébrale ;

- dosage positif des anti-AQP 4 (d'une sensibilité de $70 \%$ et d'une spécificité de $90 \%$ [2].

Le traitement de la phase aiguë repose sur l'utilisation de corticoïde à forte dose. En cas d'échec, la plasmaphérèse peut être proposée [2]. Un traitement immunomodulateur prolongé doit être associé afin de permettre la consolidation et éviter la récidive [7].

En conclusion, ce cas permet de discuter une pathologie rare, potentiellement grave mais curable, qui mérite d'être connue du médecin urgentiste. La réalisation d'une IRM médullaire, quand elle est disponible, semble être un atout diagnostique majeur, nettement supérieur à la TDM, lorsqu'une organicité métamérique ou multimétamérique est suspectée.

Conflits d'intérêts : Les auteurs déclarent ne pas avoir de liens d'intérêts.

\section{Références}

1. Frohman EM, Wingerchuk DM (2010) Clinical practice. Transverse myelitis. N Engl J Med 363:564-72

2. Trebst C, Jarius S, Berthele A, et al (2014) Update on the diagnosis and treatment of neuromyelitis optica: recommendations of the Neuromyelitis Optica Study Group (NEMOS). J Neurol 261:1-16

3. Lennon VA, Wingerchuk DM, Kryzer TJ, et al (2004) A serum autoantibody marker of neuromyelitis optica: distinction from multiple sclerosis. Lancet 364:2106-12

4. Jarius S, Paul F, Franciotta D, et al (2008) Mechanisms of disease: aquaporin-4 antibodies in neuromyelitis optica. Nat Clin Pract Neurol 4:202-14

5. Riphagen J, Modderman P, Verrips A (2010) Hiccups, nausea, and vomiting: water channels under attack! Lancet 375:954

6. Wingerchuk DM, Lennon VA, Pittock SJ, et al (2006) Revised diagnostic criteria for neuromyelitis optica. Neurology 66:1485-9

7. De Seze J, Kremer L, Collongues N (2016) Neuromyelitis optica spectrum disorder (NMOSD): a new concept. Rev Neurol $172: 256-62$ 